\title{
Persistent effects after trigeminal nerve proprioceptive stimulation by mandibular extension on rat blood pressure, heart rate and pial microcirculation
}

\author{
D. LAPI', A. COLANTUONI', C. DEL SEPPIA ${ }^{2}$, S. GHIONE ${ }^{2,3}$, \\ D. TONLORENZI ${ }^{4}$, M. BRUNELLI ${ }^{4}$, R. SCURI ${ }^{5}$ \\ 'Department of Neuroscience, "Federico II", University Medical School, University of Naples, Italy; \\ ${ }^{2}$ Institute of Clinical Physiology, National Council of Research (CNR), Pisa, Italy; \\ ${ }^{3}$ Fondazione Toscana Gabriele Monasterio - Medical and Public Health Research, Pisa, Italy; \\ ${ }^{4}$ CRISAF, Department of Physiopathology, Experimental Medicine and Public Health, \\ University of Siena, Italy; \\ ${ }^{5}$ Department of Translational Research on New Technologies in Medicine and Surgery, \\ University of Pisa, Italy
}

\begin{abstract}
A B S T R A C T
The trigemino-cardiac reflex is a brainstem reflex known to lead to a decrement in heart rate and blood pressure, whereas few data have been collected about its effects on the cerebral hemodynamic. In this study we assess the in vivo effects of trigeminal nerve peripheral stimulation by mandibular extension on pial microcirculation and systemic arterial blood pressure in rats. Experiments were performed in male Wistar rats subjected to mandibular extension obtained inserting an ad hoc developed retractor between the dental arches. Mean arterial blood pressure and heart rate were recorded and the pial arterioles were visualized by fluorescence microscopy to measure the vessel diameters before (15 minutes) during (5-15 minutes) and after (80 minutes) mandibular extension. While in control rats (sham-operated rats) and in rats subjected to the dissection of the trigeminal peripheral branches mean arterial blood pressure, heart rate and pial microcirculation did not change during the whole observation period (110 minutes), in rats submitted to mandibular extension, mean arterial blood pressure, heart rate and arteriolar diameter significantly decreased during stimulation. Afterward mean arterial blood pressure remained reduced as well as heart rate, while arteriolar diameter significantly increased evidencing a vasodilatation persisting for the whole remaining observation time. Therefore, trigeminal nerve proprioceptive stimulation appears to trigger specific mechanisms regulating systemic arterial blood pressure and pial microcirculation.
\end{abstract}

Key words

Mandibular extension $\bullet$ Trigeminal nerve $\bullet$ Pial $\bullet$ Microcirculation $\bullet$ Systemic arterial pressure

\section{Introduction}

The trigeminal nerve is the largest of the cranial nerves and provides sensory supply to the face, scalp and mucosa of nose and mouth (Brown, 1997).

Stimulation of the trigeminal receptors innervating the nose and the nasal passages is thought to pro- vide an important stimulus for the initiation of the trigeminal respiratory reflex and cardiac arrhythmias which could arise with it (Blanc et al., 1988; Schaller, 2004; Cornelius et al., 2010). These effects have been studied in animals and have been included in the named trigemino-cardiac reflex (TCR) or trigeminal depressor response (TDR) (Kumada et 
al., 1977). TCR gained much clinical attention in the form of the oculo-cardiac reflex which is the cardiac response (mainly bradycardia) associated with stimulation of the ophthalmic division of the trigeminal nerve during ocular surgeries (Blanc et al., 1983; Schaller, 2004; Schaller et al., 2008). Several lines of experimental evidence demonstrate that the TCR may occur with manipulation of all branches of the trigeminal nerve anywhere along its (intracranial or extracranial) course and in each event causes changes in heart rate (HR) and blood pressure (BP) (Butler and Jones, 1997; Schaller et al., 1999, 2008; Schaller and Graf, 2003; Graf and Schaller, 2004; Gharabaghi et al., 2006).

At cerebral level, TCR is the expression of a central neurogenic reflex leading to a rapid cerebrovascular vasodilatation generated by excitation of neurons in the rostral ventrolateral medulla oblongata. Through this physiological response, both systemic and cerebral circulation are finely regulated (Sandu et al., 2010).

We recently reported in humans that submaximal mouth opening obtained for ten minutes by applying a dilatator between the upper and lower incisor teeth, associated to partial masticatory movements, induced a long-lasting reduction of blood pressure and heart rate (Brunelli et al., 2012) by about $10 \%$. We hypothetized that this phenomenon represents a late effect of proprioceptive stimulation of the mandibular trigeminal branch.

To the best of our knowledge, this effect has not been previously described; because of its potential interest, we tried to implement an experimental animal model to reproduce this phenomenon and assess its characteristics.

In the present study we describe an in vivo model in the anesthetized rat that permits to assess the cardiovascular effects of mandibular extension previously observed in humans. In particular, we here report the effects of mandibular extension of various time durations on heart rate, systemic arterial blood pressure and pial microcirculation.

\section{Materials and methods}

\section{Experimental groups}

Male normotensive Wistar rats weighing 250-300 g (Harlan, Italy) were kept in basal conditions for 15 minutes and then randomly assigned to five groups:
1) rats not subjected to mandibular extension (ME) [Sham-Operated ( $\mathrm{SO}$ ) group, $\mathrm{n}=5$ ]; 2) rats submitted to 5 minutes-lasting $\mathrm{ME}$ (group $\mathrm{ME}_{5 \min }, \mathrm{n}=5$ ); 3) rats submitted to 10 minutes-lasting ME (group $\left.\mathrm{ME}_{10 \min }, \mathrm{n}=5\right)$; 4) rats submitted to 15 minuteslasting $\mathrm{ME}$ (group $\mathrm{ME}_{15 \min }, \mathrm{n}=5$ ); 5) rats in which the three peripheral branches of the trigeminal nerve were bilaterally cutted before being submitted to 10 minutes-lasting ME (Control group, $n=5$ ). Baseline measurements were obtained at the end of the basal condition and post-treatment measurements were obtained immediately after ME (or, in the SO group, 15 minutes after baseline observation) and were continued every 5 minutes for further 80 minutes. Mean arterial blood pressure (MABP, see below) and heart rate (HR) were constantly recorded, while the diameter changes of pial arterioles were monitored (see below) for 1 minute every 5 minutes. All data were sampled every 5 minutes for the whole observation period. HR was monitored with a Gould Windograf recorder (model 13-6615-10S, Gould, OH, USA). Data were recorded and stored in a computer for off-line analyses.

All experiments were conducted according to the Guide for the Care and Use of Laboratory Animals published by the US National Institutes of Health (NIH Publication No. 85-23, revised 1996) and to institutional rules for the care and handling of experimental animals.

\section{Animal preparation}

The rats were anesthetized with alpha-chloralose, $60 \mathrm{mg} / \mathrm{kg}$ body weight, tracheotomized, intubated, paralyzed and mechanically ventilated with room air and supplemental oxygen.

A catheter was placed in the left femoral artery for MABP recording with a Viggo-Spectramed P10E2 trasducer (Oxnard, CA, USA) and blood gases sampling with an ABL5 (Radiometer, Copenhagen, Denmark). Blood gas measurements were carried out on arterial blood samples withdrawn every 30 minutes during the whole observation period.

Another catheter was placed in the right femoral vein for injection of the fluorescent tracer [fluorescein isothiocyanate bound to dextran, molecular weight $70 \mathrm{KDa}$ (FD 70)].

Rectal temperature was monitored and maintained at $37.0 \pm 0.5^{\circ} \mathrm{C}$ with a heating stereotaxic frame, where the rats were secured. 
To observe the pial microcirculation, a cranial window $(4 \times 5 \mathrm{~mm})$ was done above the left parietal cortex (posterior $1.5 \mathrm{~mm}$ to bregma; lateral, $3 \mathrm{~mm}$ to the midline, area 5) (Ngai et al., 1988), by gently removing the dura mater. To prevent overheating of the cerebral cortex during drilling, cold saline solution was suffused on the skull. The window inflow and outflow were assured by two needles so that the brain parenchyma was continuously superfused with artificial cerebrospinal fluid (aCSF). The rate of superfusion was $0.5 \mathrm{~mL} / \mathrm{min}$ controlled by a peristaltic pump. The composition of the aCSF was: 119.0 $\mathrm{mM} \mathrm{NaCl}, 2.5 \mathrm{mM} \mathrm{KCl}, 1.3 \mathrm{mM} \mathrm{MgSO}{ }_{4} \cdot 7 \mathrm{H}_{2} \mathrm{O}, 1.0$ $\mathrm{mM} \mathrm{NaH} \mathrm{PO}_{4}, 26.2 \mathrm{mM} \mathrm{NaHCO}, 2.5 \mathrm{mM} \mathrm{CaCl}_{2}$ and $11.0 \mathrm{mM}$ glucose (equilibrated with $10.0 \% \mathrm{O}_{2}$, $6.0 \% \mathrm{CO}_{2}$ and $84.0 \% \mathrm{~N}_{2} ; \mathrm{pH} 7.38 \pm 0.02$ ) (Ngai et al., 1988; Moreau et al., 1995).

In the group that was subjected to bilateral trigeminal cutting, the three peripheral branches of the trigeminal nerve were carefully dissected after undercutting the cheek skin. Then, the skin was sutured with catgut ETHICON 2 (Pomezia, RM, Italy).

\section{Mandibular extension}

Mandibular extension (ME) was obtained by a "spring device" placed between the superior and inferior dental arches of the rat. The spring device, appropriately designed for rat, consisted of two thin layers covered with a silicone elastomer (Sylgard, Dow Corning, Midland, MI) coupled to an adjustable spring. The degree of ME was set at the maximum mouth opening, where electromyographic activity of the anterior temporalis and masseter muscles did not show any muscle fatigue. This procedure was performed for each animal submitted to ME.

Fluorescent microscopy technique and microvascular parameter assessment

Pial microcirculation was visualized with a fluorescence microscope (Leitz Orthoplan) fitted with long-distance objectives [2.5 x, numerical aperture (NA) $0.08 ; 10 \mathrm{x}$, NA $0.20 ; 20 \mathrm{x}$, NA $0.25 ; 32 \mathrm{x}$, NA $0.40]$, a $10 \mathrm{x}$ eyepiece and a filter block (Ploemopak, Leitz). Epiillumination was provided by a $100 \mathrm{~W}$ mercury lamp using the appropriate filters for FITC, and a heat filter (Leitz KG1). Pial microcirculation was televised with a DAGE MTI 300RC low-light level digital camera and recorded by a computer based frame grabber (Pinnacle DC 10 plus, Avid Technology, MA, USA).

Video images were videotaped and microvascular measurements (diameter and length) were made off-line using a computer-assisted imaging software system (MIP Image, CNR, Institute of Clinical Physiology, Pisa, Italy). The results of diameter measurements were in accord with those obtained by shearing method $( \pm 0.5 \mu \mathrm{m})$. To avoid bias due to single operator measurements, two independent "blinded" operators measured the vessel diameters. Their measurements overlapped in all cases.

The arteriolar network was mapped by stop-frame images and pial arterioles were classified according to a centripetal ordering scheme (Strahler method, modified according to diameter) (Kassab et al., 1993). Order 0 was assigned to the capillaries; thereafter the terminal arterioles were assigned to the order 1 and the vessels upstream were assigned to progressive higher orders. When two vessels of the same order joined, the parent vessel was assigned to the next higher order. If two daughter vessels were of different orders, the parent vessel retained the higher of the two orders. The procedure of the pial arterioles classification was previously reported (Lapi et al., 2008).

\section{Statistical analysis}

All data were expressed as mean \pm SEM. Data were tested for normal distribution with the KolmogorovSmirnov test. Due to the normality of distribution, one way ANOVA and Bonferroni or Dunnett's post hoc test were used. The statistical analysis was carried out by GraphPad Prism 4.0 statistical package. Statistical significance was set at $\mathrm{p}<0.05$.

\section{Results}

\section{Pial microcirculation}

Under baseline conditions pial arterioles were classified in five orders according to diameter, length and branching (Table I; Fig. 1). Capillaries, assigned to order 0 , originated from the smallest arterioles (order 1, average diameter: $16.00 \pm 0.20 \mu \mathrm{m}$, average length: $140.00 \pm 7.60 \mu \mathrm{m} ; \mathrm{n}=120$ ). The largest pial arterioles, order 5, showed diameter of 62.00 $\pm 1.30 \mu \mathrm{m}$ and length of $1169.00 \pm 108.50 \mu \mathrm{m}(\mathrm{n}$ $=16$ ). The values of diameters were significantly different between the different arteriolar orders 
Table I. - Classification of the pial arterioles in according with the Strahler's scheme.

\begin{tabular}{|c|c|c|c|c|}
\hline Order & Arterioles $(\mathrm{N})$ & Diameter $\mu \mathrm{m})$ & Length $\mu \mathrm{m})$ & Rats $(\mathrm{N})$ \\
\hline 5 & 16 & $62.0 \pm 1.3^{*}$ & $1169.0 \pm 108.5$ & 20 \\
\hline 4 & 35 & $46.0 \pm 0.6^{*}$ & $987.0 \pm 42.9$ & 20 \\
\hline 3 & 87 & $33.0 \pm 0.4^{*}$ & $481.0 \pm 12.9$ & 20 \\
\hline 2 & 148 & $24.0 \pm 0.2^{*}$ & $360.0 \pm 8.2$ & 20 \\
\hline 1 & 120 & $16.0 \pm 0.2^{*}$ & $140.0 \pm 7.6$ & 20 \\
\hline
\end{tabular}

Means values \pm standard errors of diameter and length of the pial arterioles visualized in our preparations have been reported. Pial arterioles resulted belonging to 5 orders because diameters were significantly different each other. $p<0.001$ for all pairwise comparisons.

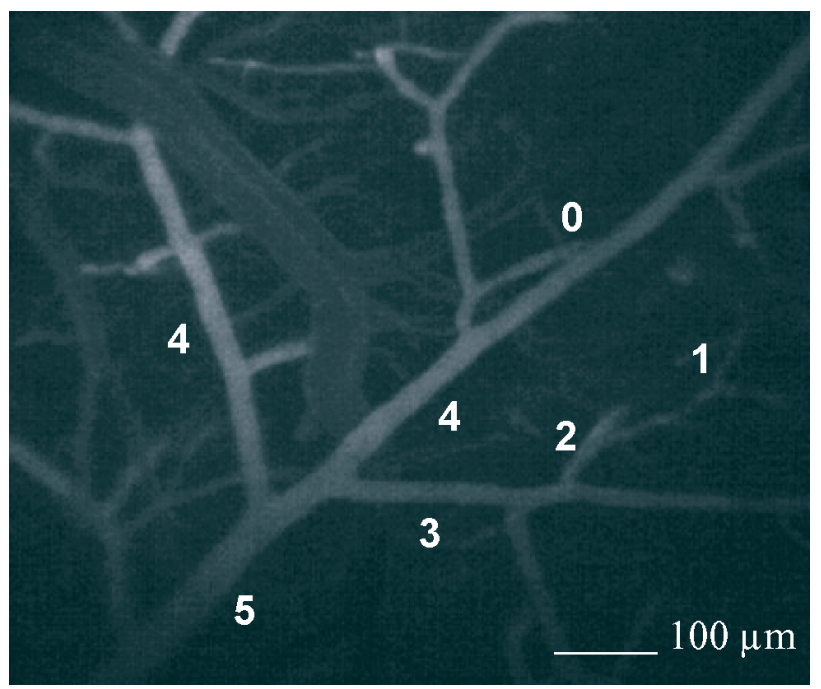

Fig. 1. - Computer-assisted image of a rat pial microvascolar network on the left parietal cortex. Within 15-20 $\mathrm{s}$ of fluorescent dextran $(70 \mathrm{kDa})$ injection, the different arteriolar orders $(5,4,3,2$ and 1 ) and capillaries (order 0) were the first vessels visualized.

(ANOVA, $\mathrm{F}_{4,405}=1468, P<0.001$; Bonferroni post hoc test, $P<0.001$ for all pairwise comparisons) (Lapi et al., 2008).

\section{Sham-Operated (SO) and control rats}

SO rats and control rats (which have the three peripheral branches of the trigeminal nerve dissected) did not show significant changes during the overall observation period (110 min) in, MABP (Fig. 2) HR (Table II) and pial arteriolar diameter for all vessel orders (see for an example of order 2 arteriole, Fig. 3).

\section{Five minutes $M E$}

\section{Effects at the end of ME}

Rats submitted to ME for 5 minutes displayed at the end of ME significant reductions in MABP, HR and arteriolar diameter. For MABP, compared to baseline $(109.9 \pm 1.07 \mathrm{mmHg})$, the value at the end of ME was $99.82 \pm 3.06 \mathrm{mmHg}$ (with a decline of $-8.24 \pm 3.36 \% ; \mathrm{F}_{17,99}=267.4, P<0.01$ ) (Fig. 2). For HR (baseline value $=321.67 \pm 3.82$ beats $/ \mathrm{min}$, Table II) the decline was of $-14.49 \pm 1.11 \%\left(\mathrm{~F}_{2,14}=173.8\right.$, $P<0.01 v s$. basal value). For arteriolar diameter (as shown in Fig. 4), all arteriolar orders exhibited a significant decrease with respect to baseline values: diameters of arterioles of order 5 (baseline: $57.00 \pm$ $1.23 \mu \mathrm{m}$ ) and of order 4 (baseline: $46.6 \pm 1.06 \mu \mathrm{m}$ ) decreased by $3.15 \pm 1.2 \%\left(\mathrm{~F}_{17,99}=45.24, P<0.01\right.$ vs. basal value $)$ and $2.8 \pm 1.0 \%\left(\mathrm{~F}_{17,99}=180.4, P<\right.$ $0.01 \mathrm{vs}$. basal value), while diameters of arterioles of order 3 (baseline: $34.2 \pm 1.06 \mu \mathrm{m}$ ), order 2 (baseline: $24.00 \pm 0.88 \mu \mathrm{m}$ ) and order 1 (basal value $=11.00 \pm$ $0.18 \mu \mathrm{m})$ decreased by $6.0 \pm 2.3 \%\left(\mathrm{~F}_{17,99}=133.9, P\right.$ $<0.01$ vs. basal value $), 5.7 \pm 2.0 \%\left(\mathrm{~F}_{17,99}=103.5, P\right.$ $<0.01 v$ s. basal value $)$ and $6.1 \pm 2.0 \%\left(\mathrm{~F}_{17,99}=28.28\right.$, $P<0.01 v s$. basal value) respectively.

\section{Effects following ME}

In the observation period following ME, MABP and HR further slightly decreased, the decrement compared to baseline, after 80 minutes being respectively by $11.57 \pm 2.65 \%(P<0.01 v s$. basal value, Fig. 2) and by $16.76 \pm 1.73 \%(P<0.01 v s$. basal value). As regards pial vessels, the vasoconstrictive response observed during $\mathrm{ME}$ was followed by a vasodilatation. In fact, order 5 and 4 arteriolar diameter significantly increased compared to baseline (by $15 \pm 3 \%$ and $15.8 \pm 2.9 \% P<0.01 v s$. basal value), while the order 3, 2 and 1 arterioles showed a diameter increase by $23 \pm 4 \%, 22.0 \pm 3.5 \%$ and $22.3 \pm$ $2.0 \%$ of baseline, respectively $(P<0.01 v s$. baseline $)$ (Fig. 4). Arteriolar dilation lasted for at least $65 \mathrm{~min}$; then all arterioles progressively recovered their basal diameter. 


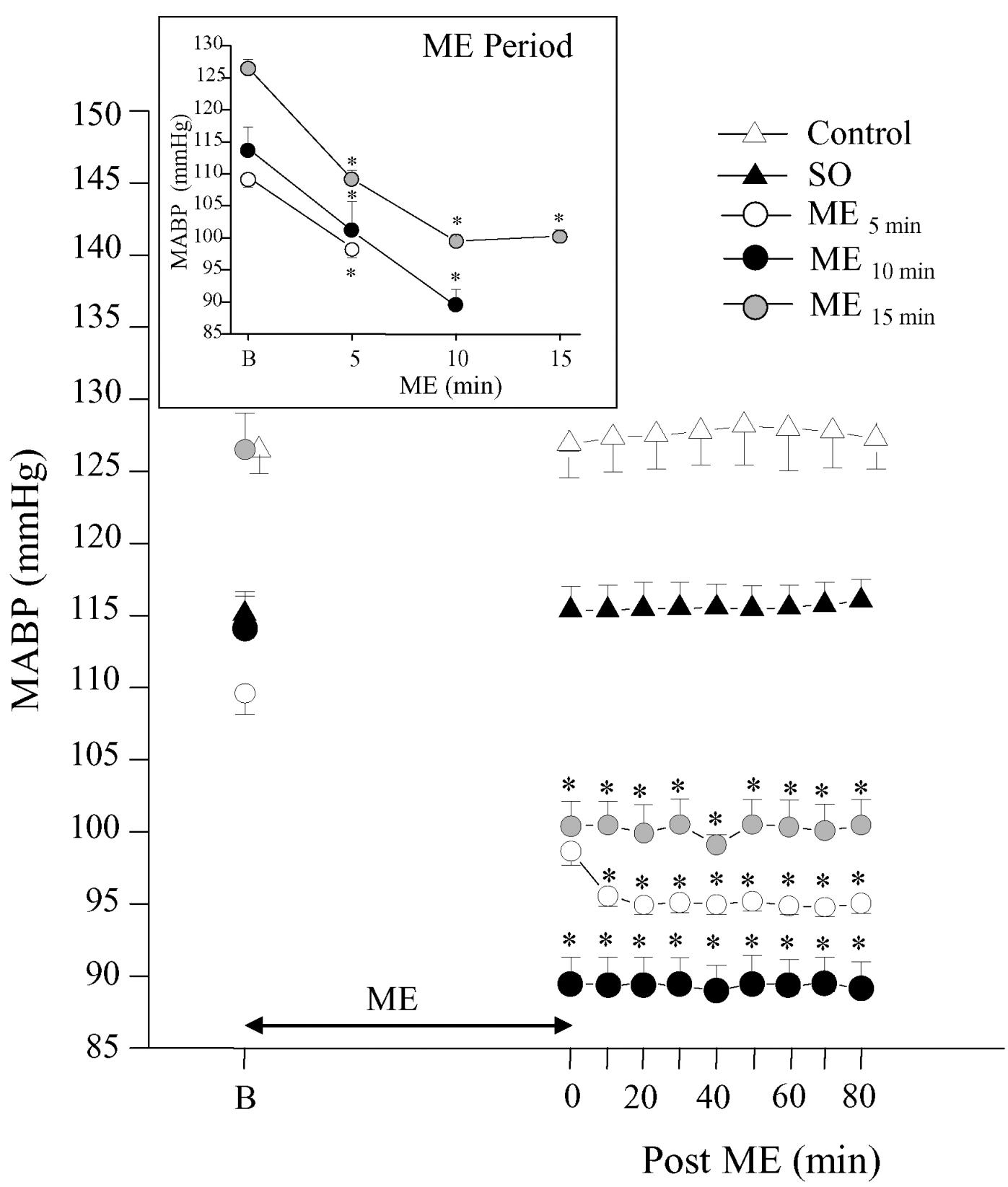

Fig. 2. - Effects of ME on MABP. In the figure the values of mean arterial blood pressure (MABP) recorded during the different experimental phases are plotted. Inset shows the changes of MABP during ME of different duration, and the graph the basal value (B) and the measurements obtained after ME. ME of different duration induced an immediate significant reduction of MABP in comparison with basal value lasting for the whole observation period after ME (*, ANOVA for repeated measures, and Dunnett's post hoc test vs. baseline value, $P<0.01$ ). On the contrary, no changes in MABP values have been detected in Control and SO rats during the whole observation period.

\section{Ten minutes $M E$}

\section{Effects during and at the end of ME}

When ME was extended to 10 minutes MABP and HR further declined compared to values recorded after 5 minutes (for MABP see inset in Fig. 2; for HR data not shown). The value of MABP at the end of ME was reduced by $21.64 \pm 0.26 \%$ of baseline $\left(\mathrm{F}_{18,99}=376.1, P<0.01\right.$ vs. basal value $=113.94 \pm$ $2.34 \mathrm{mmHg}$ ) and HR decreased by $21.66 \pm 0.25 \%$ of basal value $\left(\mathrm{F}_{2,14}=1106.0, P<0.01 v s\right.$. basal value $=321.67 \pm 1.44$ beats $/ \mathrm{min})$. For arteriolar diameter Fig. 5 shows that, all arteriolar diameters decreased 
Table II. - Changes of HR after mandibular extension.

\begin{tabular}{|l|c|c|c|}
\hline & Basal value & At the end of ME & At the end of the post ME period (80 min) \\
\hline Control & $325.7 \pm 0.4$ & $324.7 \pm 0.4$ & $323.0 \pm 0.71$ \\
\hline Sham-operated & $330.0 \pm 25$ & $332.0 \pm 23.2$ & $331.0 \pm 20$ \\
\hline $\mathrm{ME}_{5 \min }$ & $321.7 \pm 3.8$ & $275.0 \pm 2.5^{*}$ & $267.7 \pm 2.9^{*}$ \\
\hline $\mathrm{ME}_{10 \mathrm{~min}}$ & $321.7 \pm 1.4$ & $252.0 \pm 1.0^{*}$ & $259.7 \pm 1.0^{*}$ \\
\hline $\mathrm{ME}_{15 \mathrm{~min}}$ & $333.0 \pm 1.9$ & $270.7 \pm 3.9^{*}$ & $273.0 \pm 0.41^{*}$ \\
\hline $\begin{array}{l}\text { Mean values } \pm \text { standard errors of HR have been reported. HR has been expressed as beats/min and all value have been compared with the } \\
\text { basal value in all experimental groups. " } \mathrm{p}<0.01 \text { vs. basal value }\end{array}$ \\
\hline
\end{tabular}

after ME for 5 minutes and did not further vary after 10 minutes. In particular, in order 5 (basal value $=$ $64.0 \pm 1.1 \mu \mathrm{m})$ and 4 (basal value $=45.2 \pm 1.06 \mu \mathrm{m})$ vessels, at the end of $\mathrm{ME}$ diameters decreased by $8.2 \pm 3.0 \%\left(\mathrm{~F}_{18,99}=245.7, P<0.01\right)$ and $7.4 \pm 1.8 \%$ of basal value $\left(\mathrm{F}_{18,99}=271.5, P<0.01 v s\right.$. baseline $)$ respectively, while order 3 (basal value $=31.00 \pm$ $0.77 \mu \mathrm{m}), 2$ (basal value $=24.80 \pm 0.74 \mu \mathrm{m})$ and 1 (basal value $=16.50 \pm 0.35 \mu \mathrm{m})$ arterioles constricted by $18.0 \pm 2.0 \%\left(\mathrm{~F}_{18,99}=681.1, P<0.01 v s\right.$. baseline $), 19.5 \pm 3.0 \%\left(\mathrm{~F}_{18,99}=391.0, P<0.01\right.$ vs. basal value $)$ and $20.0 \pm 2.2 \%$ of basal value $\left(\mathrm{F}_{18.99}=\right.$ 328.0, $P<0.01 v s$. basal value), respectively.

\section{Effects following ME}

In the observation period following ME, MABP and HR remained stably reduced for the whole observation time $(P<0.01 v s$. basal value $)$ and essentially equal to the value attained at the end of 10 minutes ME $(89.28 \pm 1.87 \mathrm{mmHg}, 252.0 \pm 1.0$ beats $/ \mathrm{min}$ respectively; $P<0.01$ vs. basal value), see Fig. 2 for MABP and Table II for HR. In all pial arteriolar orders, the vasoconstrictive response observed during ME was followed by a marked vasodilatation (Fig. 5) lasting for the whole observation period. At the end of the observation period, in vessels of order 5 and 4 the increase of diameters was by $21.8 \pm 3.2 \%$ and $22.3 \pm 2.0 \%$ of basal value $(P<0.01 \mathrm{vs}$. basal value) respectively, while in order 3,2 and 1 vessels the diameter increase was higher than in the larger vessels $(30.0 \pm 2.5 \%, 31.4 \pm 2.3 \%$ and $30.9 \pm 2.5 \%$ of basal value, respectively, $P<0.01 v s$. basal value).

\section{Fifteen minutes $M E$}

\section{Effects during and at the end of ME}

In the observation period following with during ME, MABP and HR remained stably reduced for the whole observation time $\left(\mathrm{F}_{19,99}=215.4, P<0.01\right.$ vs. basal value and $\mathrm{F}_{2,14}=197.1, P<0.01$ vs. basal value, respectively) and essentially equal to the value attained at the end of 10 minutes ME (for MABP see inset in Fig. 2; for HR data not shown). At the end of ME, MABP decreased by $20.71 \pm 0.48 \%$ of basal value $(P<0.01 v s$. basal value) and HR reduced by $18.30 \pm 1.40 \%$ of basal value, $(P<0.01 v s$. basal value). In pial vessels, no further vasoconstriction was found when ME was extended to 15 minutes. At the end of ME, order 5 (basal value $=60.00 \pm 0.98$ $\mu \mathrm{m})$ and 4 (basal value $=47.00 \pm 0.88 \mu \mathrm{m}$ ) arterioles showed a diameter reduction by $5.0 \pm 1.9 \%\left(\mathrm{~F}_{19.99}=\right.$ 3204.0, $P<0.01 v s$. basal value) and $7.9 \pm 2.0 \%$ of basal value $\left(\mathrm{F}_{19,99}=1031, P<0.01\right.$ vs. basal value $)$ respectively, while order 3 (basal value $=33.30 \pm$ $0.71 \mu \mathrm{m}), 2$ (basal value $=22.50 \pm 0.63 \mu \mathrm{m})$ and 1 (basal value $=14.00 \pm 0.53 \mu \mathrm{m})$ vessels constricted by $17.1 \pm 2.0 \%\left(\mathrm{~F}_{19,99}=5037.0, P<0.01 v s\right.$. basal value $), 19.1 \pm 2.2 \%\left(\mathrm{~F}_{19,99}=3101.0, P<0.01 \mathrm{vs}\right.$. basal value) and $20.0 \pm 2.0 \%$ of basal value respectively $\left(\mathrm{F}_{19,99}=5086, P<0.01 \mathrm{vs}\right.$. basal value) (Fig. 6).

\section{Effects following ME}

In the observation period following ME, MABP and HR remained stably reduced for the whole observation time $(P<0.01$ vs. basal value $)$ and essentially equal to the value attained at the end of ME, see Fig. 2 for MABP and Table II for HR.

As for 10 minutes ME, also after 15 minutes ME, in all pial arteriolar orders, the vasoconstrictive response observed during $\mathrm{ME}$ was followed by a marked vasodilatation (Fig. 5) that lasted for the whole observation period. Order 5 and 4 vessels diameter increased by $15.7 \pm 2.0 \%$ and $16.2 \pm 1.8 \%$ of basal value, respectively $(P<0.01 \mathrm{vs}$. basal value), while order 3,2 and 1 vessels dilated by 30.9 $\pm 2.5 \%, 30.7 \pm 1.8 \%$ and $32.1 \pm 2.5 \%$ of basal value, respectively $(P<0.01 v s$. basal value $)$. 


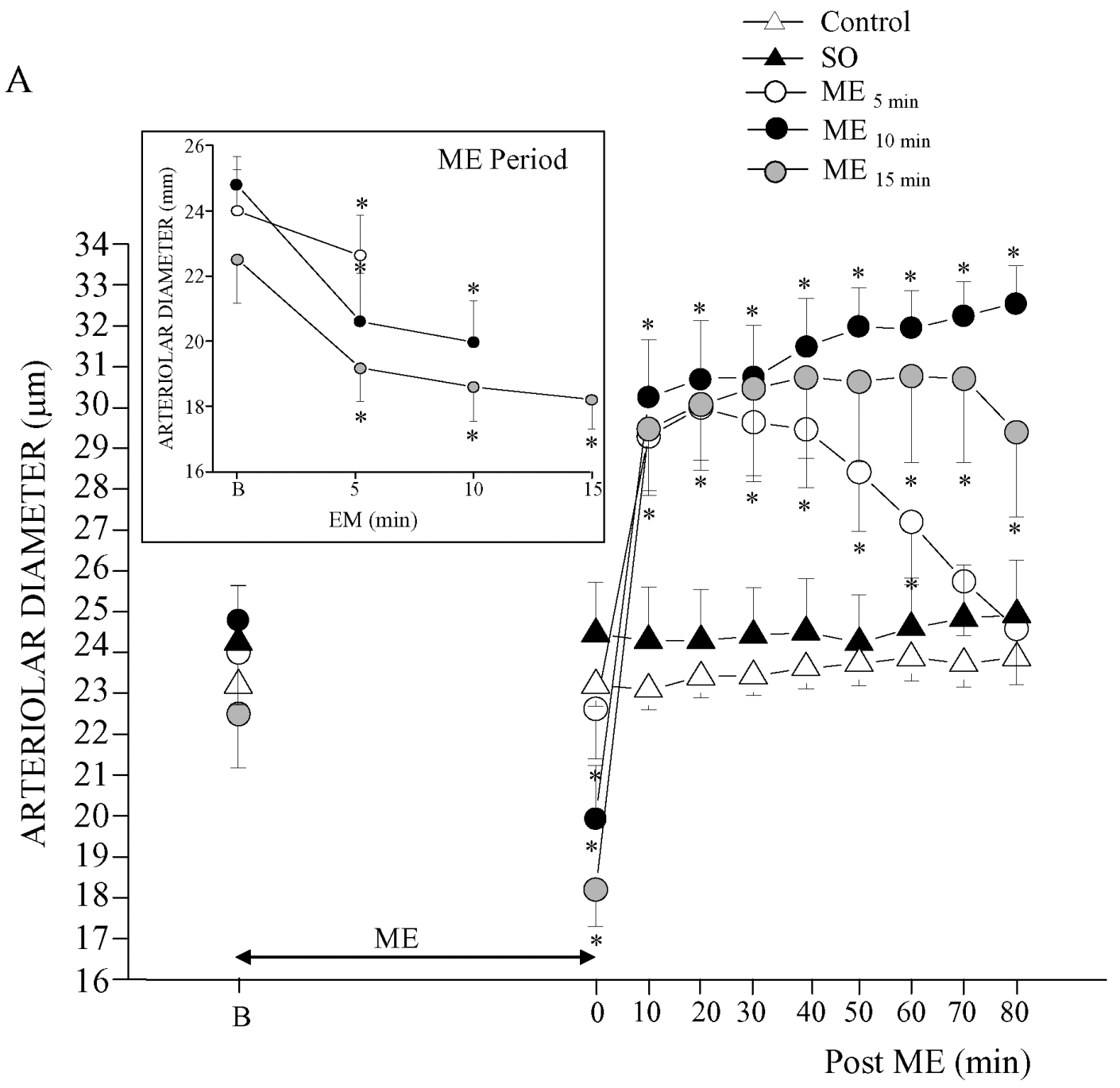

$\mathrm{B}$

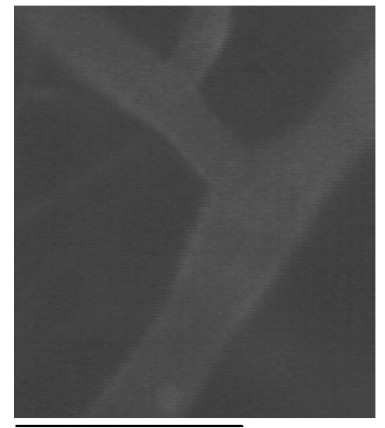

$50 \mu \mathrm{m}$

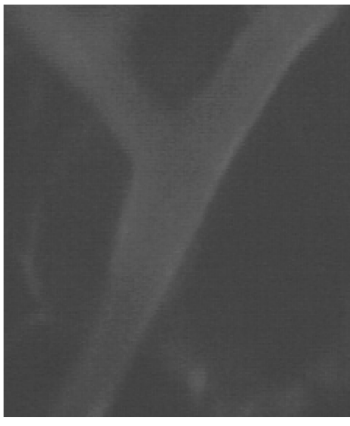

DURING ME

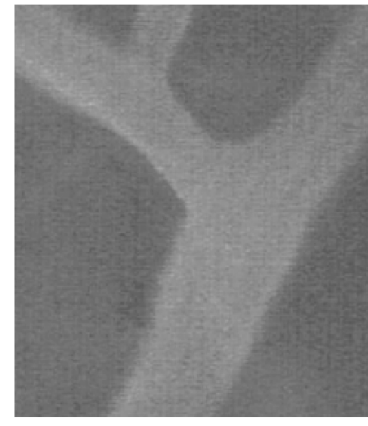

POST ME

Fig. 3. - Effects of ME on diameter of order 2 arterioles. (A) The values of the diameters of order 2 arterioles have been measured during the different experimental phases. In the inset the changes of diameters during ME of different duration have been plotted showing a significant time-dependent decrease ( ${ }^{*}$, ANOVA for repeated measures and Dunnett's post hoc test vs. basal value, $P<0.001$ ). The graph shows an increase of diameters soon after ME and a time-dependent duration of this dilatation. No changes of diameter values have been detected in Control and SO rats during the whole observation period. (B) The images show an order 2 arteriole in basal conditions at 5 minutes during 10 minutes $\mathrm{ME}$ and at 30 minutes after 10 minutes ME. 
A

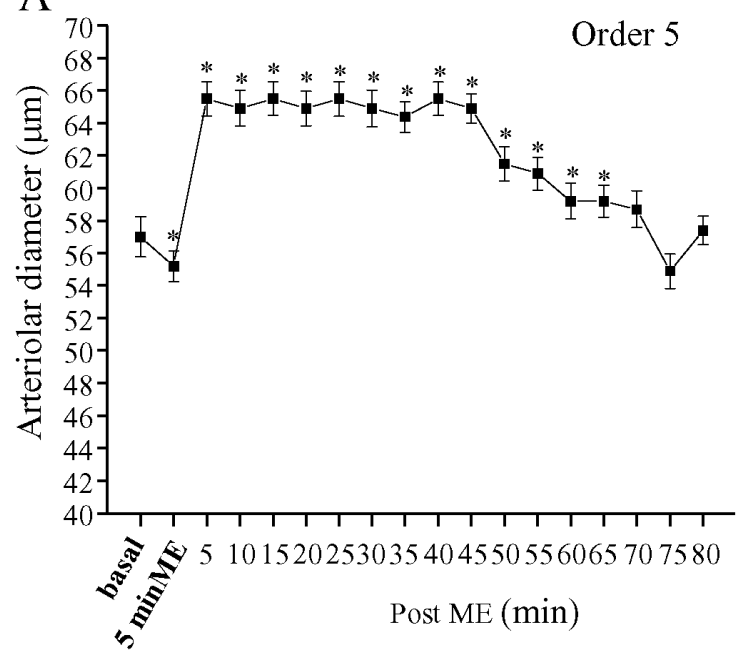

C

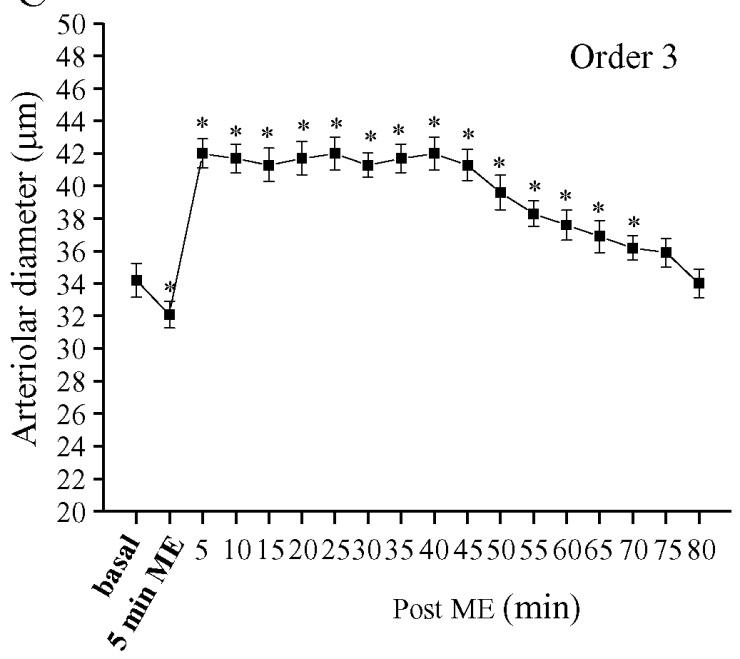

E

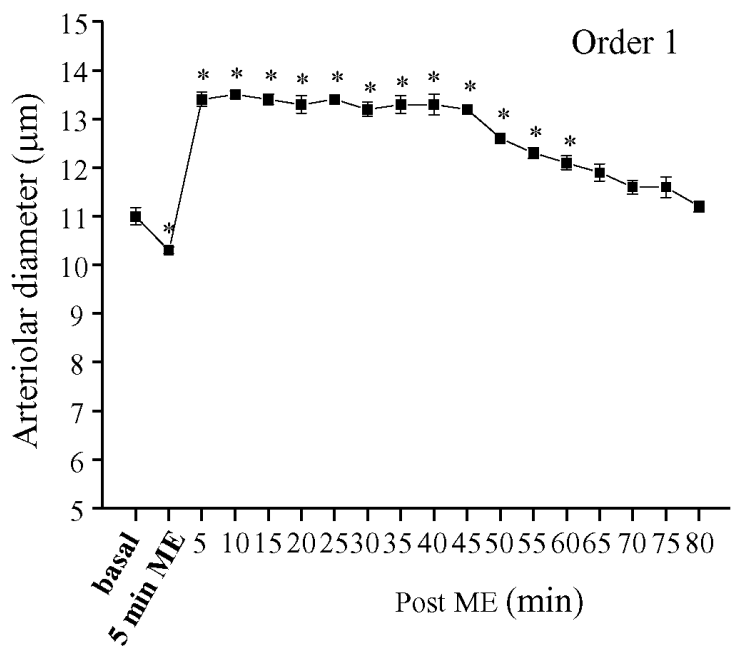

$\mathrm{B}$

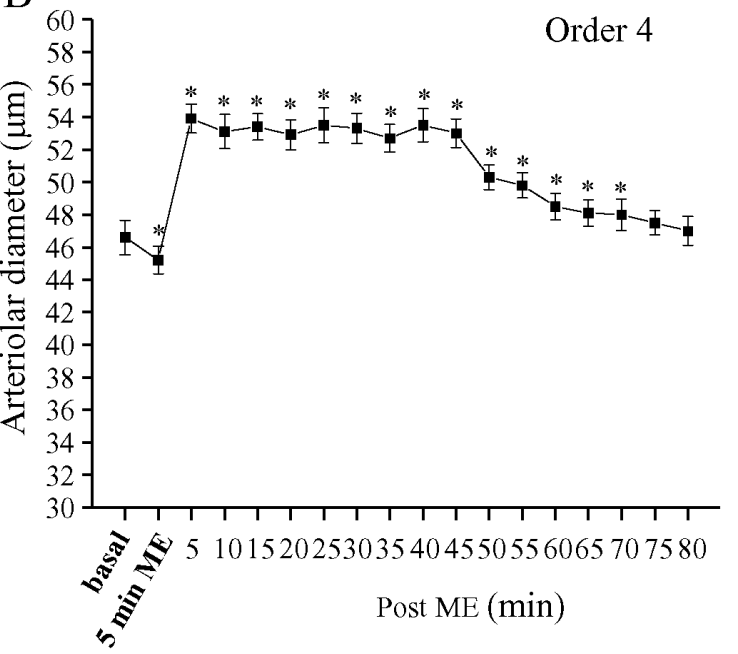

D

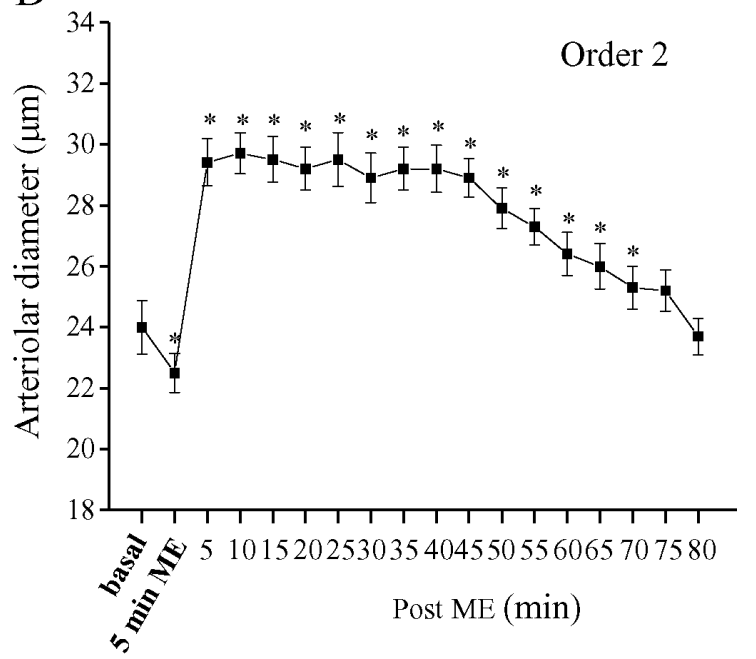

Fig. 4. - Effects of 5 minutes ME on arteriolar diameters. In the figure the value of diameters of order 5 (A), 4 (B), 3 (C), 2 (D) and 1 (E) arterioles have been plotted. The one way ANOVA for repeated measures and the Dunnett's post hoc test carried out for all arteriolar orders showed a significant reduction of diameters during ME followed by a significant increase after ME which lasted for at least 60 minutes (" indicate $P<0.05$ vs. basal value). 
A

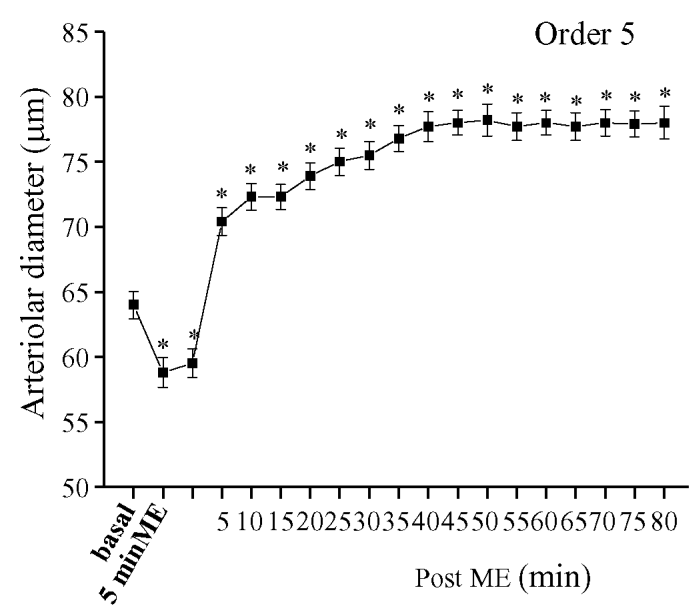

$\mathrm{C}$

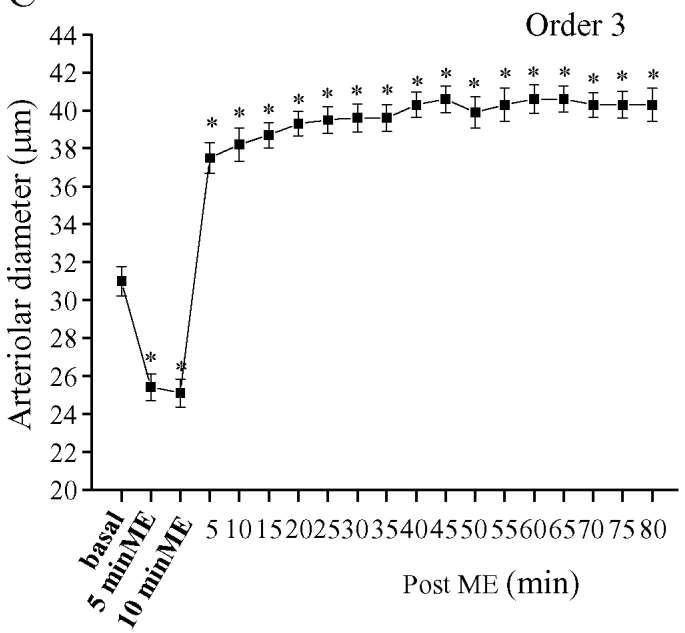

$\mathrm{E}$

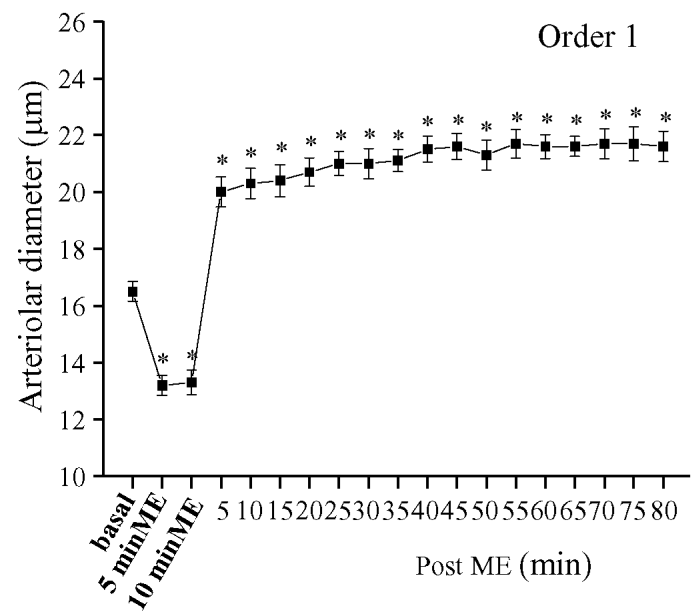

$\mathrm{B}$

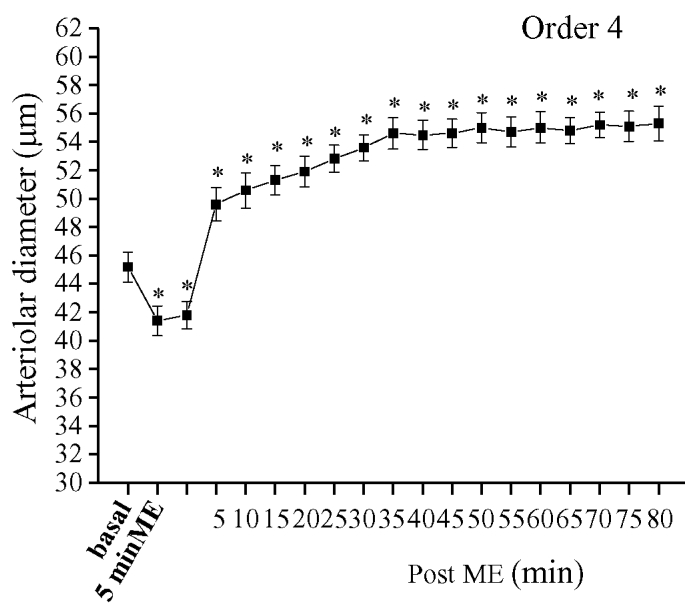

D

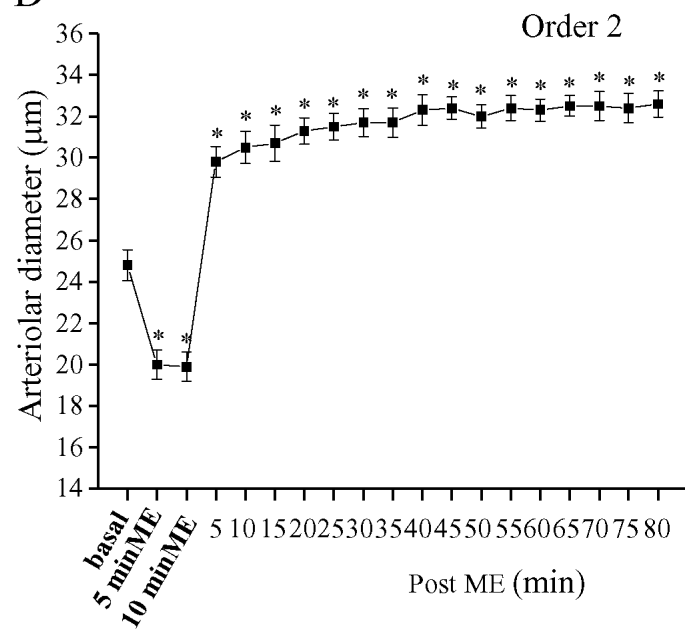

Fig. 5. - Effects of 10 minutes ME on arteriolar diameters. In the figure the value of diameters of order 5 (A), 4 (B), 3 (C), 2 (D) and 1 (E) arterioles have been plotted. The one way ANOVA for repeated measures and the Dunnett's post hoc test carried out for all arteriolar orders showed a significant reduction of diameters during the first 5 minutes ME which remained essential equal for the further 5 minutes ME, followed by a significant vasodilation which lasted for the whole observation period after ME ( ${ }^{*}$ indicate $P<0.01$ vs. basal value). 
A

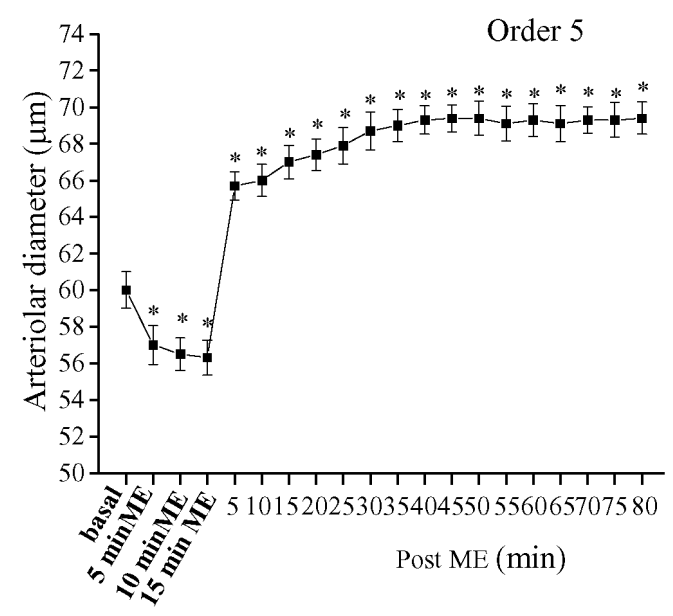

C

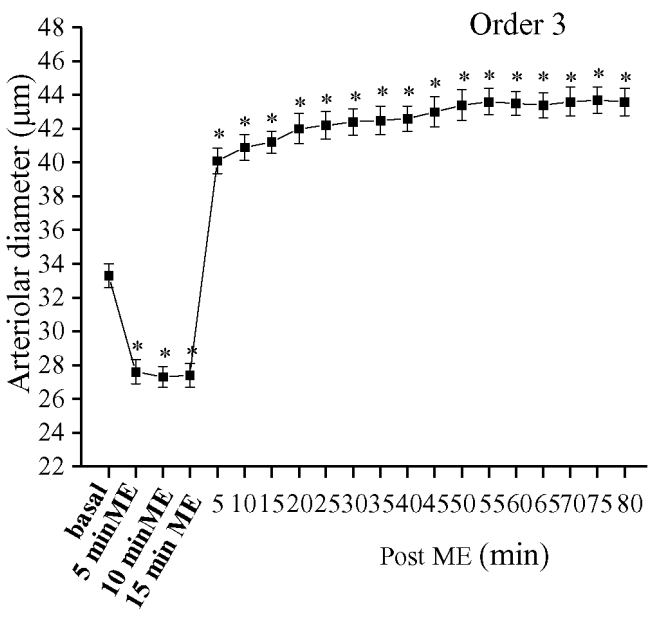

E

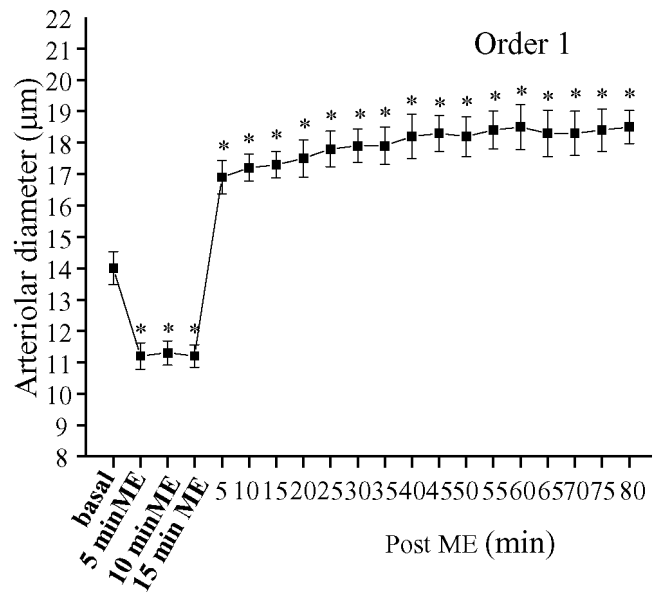

B

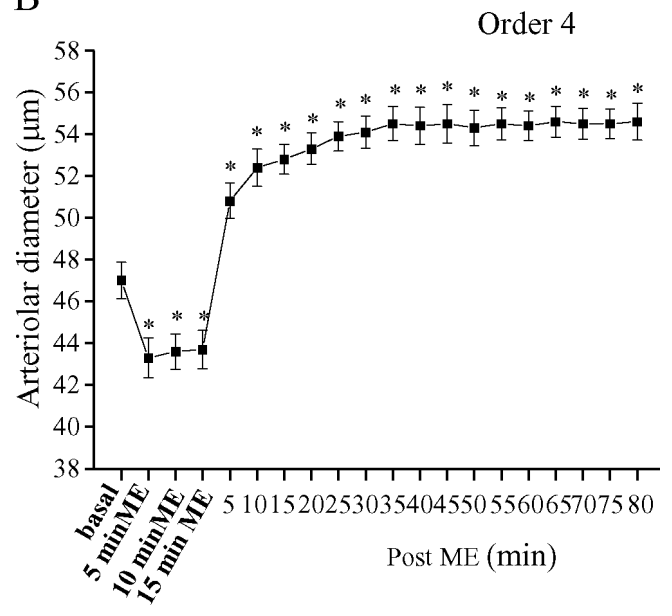

D

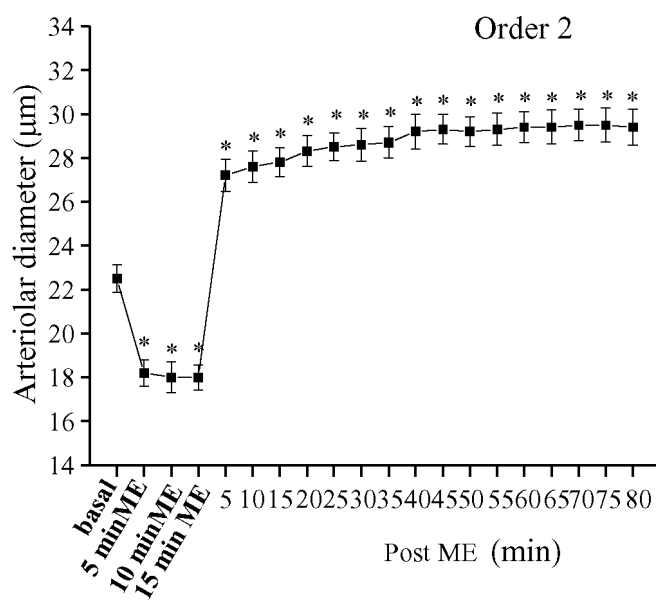

Fig. 6. - Effects of 15 minutes ME on arteriolar diameters. In the figure the value of diameters of order 5 (A), 4 (B), 3 (C), 2 (D) and 1 (E) arterioles have been plotted. The one way ANOVA for repeated measures and the Dunnett's post hoc test carried out for all arteriolar orders showed a significant reduction of diameters during the first 5 minutes ME which remained essential equal for the further 10 minutes ME, followed by a significant vasodilation which lasted for the whole observation period after ME ( ${ }^{*}$ indicate $P<0.01$ vs. basal value). 


\section{Discussion}

This study carried out in an experimental animal model confirms our findings previously reported in humans (Brunelli et al., 2012) that passive mandibular extension for 10 minutes is followed by a significant, prolonged and stable reduction of arterial blood pressure and heart rate lasting for at least 80 minutes.

In this study two distinct phases of the cardiovascular response to $\mathrm{ME}$ are evident: a first response that is concurrent to $\mathrm{ME}$ and a subsequent prolonged response that initiates after ME. Both these responses are abolished by cutting the trigeminal nerve, indicating that trigeminal afferents are involved.

In addition, the trigeminal nerve stimulation triggered by $\mathrm{ME}$ induced a biphasic response of pial arterioles which exhibited a decrease in diameter during ME followed by a pronounced increase in the post ME observation time.

The time course of the parameters studied is interesting and complex and may be described as follow. As regards MABP and $\mathrm{HR}$, the duration of ME was found to affect both the responses concomitant to ME and following ME. The response concomitant to $\mathrm{ME}$ was characterized by a decrease of MABP and HR for stimulation length increasing up to 10 minutes, without further decline thereafter. For the response following ME, a stimulus duration for at least 10 minutes was required to obtain a maximal and persistent decrement of MABP and HR. For ME duration of only 5 minutes, the decrement of MABP and $\mathrm{HR}$ after ME was lesser and less persistent than for 10 minutes-lasting ME whereas for 15 minuteslasting ME no further changes were observed.

In the pial microcirculation, the phenomenon was even more complex. The response concomitant to ME was characterized by a vasoconstriction that was already fully developed after 5 minutes and that persisted unchanged for more prolonged ME up to 15 minutes. On the other hand, following ME, an overshooting vasodilatation is observed with a time course that apparently depended on the length of ME. For 5 minutes-lasting ME the vasodilator effect persisted for about 60 minutes and then gradually subsided in the following 20 minutes, whereas for ME duration over 5 minutes the vasodilation persisted for the entire observation period.

The biphasic response of pial circulation to trigeminal nerve stimulation by ME is somewhat surprising.
In fact, pial microcirculation and systemic arterial blood pressure are in general strictly related, and pial vessels autoregulate their diameter in order to maintain adequate cerebral blood flow despite frequent changes in systemic arterial blood pressure. In particular, a systemic blood pressure decrease is usually accompanied by a vasodilation of pial arterioles. However, in our model a reduction in MABP during ME was associated to a vasoconstriction (rather than a vasodilatation), followed by a prolonged vasodilation after removing ME. It is possible to hypothesize that the vasoconstriction during $\mathrm{ME}$ is due to the activation of trigeminal proprioceptive afferents causing release of vasoconstrictor mediators, but further investigations are required to better clarify this phenomenon (Brian et al., 1996).

On the contrary, the persistent vasodilation associated to the reduction in MABP following ME is a more common event described in several animal models (Mchedlishvili, 1980; Florence and Seylaz, 1992). For example, a study carried out on anaesthetized cats demonstrated that the electrical stimulation of trigeminal ganglion induced a blood pressure decrease accompanied by a marked dilation of ipsilateral common carotid artery and bilateral dilation of middle meningeal vascular bed inducing an increase in dural circulation (Lambert et al., 1997). Nevertheless, up to date, the mechanisms underlying the vasodilation of cerebral vessels associated to trigeminal stimulation are still poorly known. A vasodilatative response to trigeminal stimulation could be triggered by a decrease in sympathetic nerve discharge or by a release of endothelium-derived vasodilators, such as nitric oxide and EDHF (Wahlm et al., 1993; Golding et al., 2002; Okamura et al., 2002; Toda et al., 2009). Further studies are needed to identify the mechanism(s) involved. In this regard, previously, Lindauer and coworkers (1999) showed that an increase of neuronal activity due to trigeminal nerve stimulation was correlated to neuronal nitric oxide release and in this case the nitric oxide effects mainly involved the arterioles adjacent to neuronal pool activated, i.e. the arterioles of lower order.

In conclusion, our data indicate that trigeminal nerve proprioceptive stimulation by mandibular extension induces a hypotensive response similar to the one we observed in the human (Brunelli et al., 2012) and a characteristic regulation of pial arteriolar tone triggered by specific mechanisms. 
The finding that in the normotensive range there are an important hypotensive response and a specific regulation of pial microcirculation makes it interesting to extend our investigations to models of hypertensive rats in order to collect data useful in clinical practice.

\section{References}

Blanc V.F., Jacob J.L., Milot J., Cyrenne L. The oculorespiratory reflex revisited. Can. J. Anaesth., 35: 468-472, 1988.

Blanc V.F., Hardy J.F., Milot J., Jacob J.L. The oculocardiac reflex: a graphic and statistical analysis in infants and children. Can. J. Anaesth., 30: 360$369,1983$.

Brian J.E., Faraci F.M., Heistad D.D. Recent insights into the regulation of cerebral circulation. Clin. Exp. Pharmacol. Physiol., 23: 449-457, 1996.

Brown J.A. The trigeminal complex. Anatomy and physiology. Neurosurg. Clin. N. Am., 8: 1-10, 1997.

Brunelli M., Coppi E., Tonlorenzi D., Del Seppia C., Lapi D., Colantuoni A., Scuri R., Ghione S. Prolonged hypotensive and bradycardic effects of passive mandibular extension: evidence in normal volunteers. Arch. Ital. Biol., 150: 231-237, 2012.

Butler P.J. and Jones D.R. Physiology of diving of birds and mammals. Physiol. Rev., 77: 837-899, 1997.

Cornelius J.F., Sadr-Eshkevari P., Arasho B.D., Sandu N., Spiriev T., Lemaitre F., Schaller B. The trigemino-cardiac reflex in adults: own experience. Expert Rev. Cardiovasc. Ther., 8: 895-898, 2010.

Florence G. and Seylaz J. Rapid autoregulation of cerebral blood flow: a laser-Doppler flowmetry study. J. Cereb. Blood Flow Metab., 12: 674-680, 1992.

Gharabaghi A., Koerbel A., Samii A., Kaminsky J., von Goesseln H., Tatagiba M., Samii M. The impact of hypotension due to the trigeminocardiac reflex on auditory function in vestibular schwannoma surgery. J. Neurosurg., 104: 369-375, 2006.

Golding E.M., Marrelli S.P., You J., Bryan R.M. Endothelium-derived hyperpolarizing factor in the brain: a new regulator of cerebral blood flow? Stroke, 33: 661-663, 2002.

Graf R., Schaller B. "Natural" tolerance in hibernators: can we learn from physiological preconditioning against ischemic or hypoxic brain injury?
In: Schaller B, (Ed.) Ischemic Tolerance of the Brain, New York, Nova Science Publishers, 2004, pp. 1-15.

Kassab G.S., Rider C.A., Tang N.J., Fung Y.C. Morphometry pig coronary arterial trees. Am. J. Physiol., 265: H350-H365, 1993.

Kumada M., Dampney R.A., Reis D.J. The trigeminal depressor response: a novel vasodepressor response originating from the trigeminal system. Brain Res., 119: 305-326, 1977.

Lambert G.A., Michalicek J., Regaglia F. Responses of the dural circulation to electrical stimulation of the trigeminal ganglion in the cat. Clin. Exp. Pharmacol. Physiol., 24: 377-390, 1997.

Lapi D., Marchiafava P.L., Colantuoni A. Geometric characteristics of arterial network of rat pial microcirculation. J. Vasc. Res., 45: 69-77, 2008.

Lindauer U., Megow D., Matsuda H., Dirnagl U. Nitric oxide: a modulator, but not a mediator, of neurovascular coupling in rat somatosensory cortex. Am. J. Physiol., 277: H799-H811, 1999.

Mchedlishvili G. Physiological mechenisms controlling cerebral blood flow. Stroke, 3: 240-248, 1980.

Moreau P., Takasa H., Küng CF., van Rooijen M.M., Schaffner T., Lüscher T.F. Structure and function of the rat basilar artery during chronic nitric oxide synthase inhibition. Stroke, 26: 19221928, 1995.

Ngai A.C., Ko K.R., Morii S., Winn H.R. Effect of sciatic nerve stimulation on pial arterioles in rats. Am. J. Physiol., 254: H133-H139, 1988.

Okamura T., Ayajiki K., Fujioka H., Shinozaki K., Toda N. Neurogenic cerebral vasodilation mediated by nitric oxide. Jpn. J. Pharmacol., 88: 32-38, 2002.

Sandu N., Cornelius J., Filis A., Nöthen C., Rasper J., Kulinsky V.I., Schaller B.J. Cerebral hemodynamic changes during the trigeminocardiac reflex: description of a new animal model protocol. Scientific World Journal, 20: 1416-1423, 2010.

Schaller B. Trigeminocardiac reflex. A clinical phenomenon or a new physiological entity? J. Neurol., 6: 658-665, 2004.

Schaller B.J., Filis A., Buchfelder M. Trigeminocardiac reflex in humans initiated by peripheral stimulation during neurosurgical skull-base operations. Its first description. Acta Neurochir. (Wien), 150: 715-717, 2008.

Schaller B. and Graf R. Hypothermia and stroke: the pathophysiological background. Pathophysiology, 10: 7-35, 2003. 
Schaller B., Probst R., Strebel S., Gratzl O. Trigeminocardiac reflex during surgery in the cerebellopontine angle. J. Neurosurg., 90: 215-220, 1999.

Toda N., Ayajiki K., Okamura T. Cerebral blood flow regulation by nitric oxide: recent advances. Pharmacol. Rev., 61: 62-97, 2009.
Wahl M. and Schilling L. Regulation of cerebral blood flow - a brief review. Acta Neurochir. Suppl. (Wien), 59: 3-10, 1993. 